\title{
Depressive Symptoms Negatively Impact Montreal Cognitive Assessment Performance: A Memory Clinic Experience
}

\author{
Mervin Blair, Kristy Coleman, Sarah Jesso, Véronique Desbeaumes Jodoin, \\ Kathy Smolewska, Erin Warriner, Elizabeth Finger, Stephen H. Pasternak
}

\begin{abstract}
Objective: The Montreal Cognitive Assessment (MoCA) is a general cognitive screening tool that has shown sensitivity in detecting mild levels of cognitive impairment in various clinical populations. Although mood dysfunction is common in referrals to memory clinics, the influence of mood on the MoCA has to date been largely unexplored. Method: In this study, we examined the impact of mood dysfunction on the MoCA using a memory clinic sample of individuals with depressive symptoms who did not meet criteria for a neurodegenerative disease. Results: Half of the group with depressive symptoms scored below the MoCA-suggested cutoff for cognitive impairment. As a group, they scored below healthy controls, but above individuals with Alzheimer's disease and frontotemporal dementia. A MoCA subtask analysis revealed a pattern of executive/attentional dysfunction in those with depressive symptoms. Conclusions: This observed negative impact of depressive symptomatology on the MoCA has interpretative implications for its utility as a cognitive screening tool in a memory clinic setting.
\end{abstract}

RÉSUMÉ: Les symptômes de dépression ont un impact négatif sur les résultats du Montreal Cognitive Assessment test : expérience d'une clinique de la mémoire. Objectif: Le Montreal Cognitive Assessment (MoCA) est un outil de dépistage cognitif général qui est sensible pour détecter des niveaux légers d'atteinte cognitive dans des populations cliniques variées. Bien qu'une dysfonction de l'humeur soit fréquente chez les patients référés à des cliniques de la mémoire, l'influence de l'humeur sur le MoCA n'a encore jamais été explorée. Méthode: Nous avons examiné l'impact d'un trouble de l'humeur sur le MoCA chez un échantillon de patients d'une clinique de la mémoire présentant des symptômes de dépression qui ne rencontraient pas les critères diagnostiques d'une maladie neurodégénérative. Résultats: La moitié du groupe présentant des symptômes de dépression avait un score sous le niveau suggéré par le MoCA pour le diagnostic d'une atteinte cognitive. En tant que groupe, leur score était inférieur à celui des témoins en bonne santé, mais supérieur à celui des patients atteints de la maladie d'Alzheimer ou de démence fronto-temporale. L'analyse des sous-tâches a montré un profil de dysfonction exécutive/de l'attention chez ceux qui présentaient des symptômes dépressifs. Conclusions: L'impact négatif de la symptomatologie dépressive sur le MoCa que nous avons observé a des implications sur l'interprétation de ce test concernant son utilité comme outil de dépistage cognitif dans le contexte d'une clinique de la mémoire.

Keywords: Alzheimer's disease, depression, frontotemporal dementia, executive function, memory, MoCA

doi:10.1017/cjn.2015.399

Can J Neurol Sci. 2016; 43: 513-517

The Montreal Cognitive Assessment (MoCA) was developed as a cognitive screening tool to help practitioners identify individuals with mild cognitive impairment. ${ }^{1}$ It has since shown sensitivity in detecting cognitive impairment in a number of other clinical populations (e.g. cerebrovascular disease, Parkinson's disease, Huntington's disease, brain tumors, epilepsy). ${ }^{2}$ Common to these various populations is a high prevalence of mood dysfunction. ${ }^{3-5}$ However, the influence of mood symptomatology, particularly depressive symptoms, on the MoCA remains unclear and represents an important area of investigation, especially because individuals presenting with depressive symptoms are common among memory clinic referrals. ${ }^{6}$

Both recent and a lifetime history of depressive symptomatology represent an increased risk factor for cognitive impairment and dementia. ${ }^{7-11}$ In human and animal studies, depressive symptomatology has been linked to abnormal function, reduced volumes, and decreased connectivity in various brain regions (e.g. prefrontal cortex, amygdala). ${ }^{12-14}$ In line with this, depressive symptomatology has been linked to deficits in various cognitive domains, ${ }^{10,15}$ particularly executive functions, and dysfunction in associated brain regions. ${ }^{16-18}$

From the Department of Cognitive Neurology, Parkwood Institute, London, Ontario, Canada (MB, KC, SJ, EF, SFP); Université du Québec à Montréal, Montréal, Québec, Canada (VDJ); Hamilton Health Sciences, Hamilton, Ontario, Canada (KS, EW); Department of Clinical Neurological Sciences, Schulich School of Medicine and Dentistry, Western University, London, Ontario, Canada (EF, SHP); Robarts Research Institute, London, Ontario, Canada (SHP)

Received May 26, 2015. Final Revisions Submitted November 6, 2015. Correspondence to: Mervin Blair, Parkwood Institute, 550 Wellington Road, London, Ontario, Canada, N6C 0A7, Email: mer.blair1@gmail.com 
Consequently, the impact of mood symptoms on a highly sensitive global cognitive tool, such as the MoCA, may be important to clarify differential diagnostic issues, especially in family practice and memory clinic settings where full neuropsychological batteries are not readily available. However, at this point our understanding of the impact of mood symptoms on the MoCA is limited with few exceptions, primarily involving community as opposed to clinical samples. ${ }^{19-21}$ Therefore, in the current study, we examined whether depressive symptoms impacted MoCA performance by employing a memory clinic sample of individuals with depressive symptoms, with the prediction that they would demonstrate impaired scores on the overall MoCA as well as MoCA subtasks, particularly those reflecting executive/attentional domains.

\section{MeTHODS}

\section{Participants}

Hospital records were reviewed for patients cognitive test scores on the $\mathrm{MoCA}^{1}$ at the time of their initial consultation to the Cognitive Neurology and Alzheimer's Research Centre at Parkwood Institute. Charts obtained ranged from 2008 (when the MoCA was added to our cognitive screening battery) to April 2014. We included individuals with depressive symptoms as well as individuals diagnosed with Alzheimer's disease or frontotemporal degeneration (FTD) and healthy volunteers.

The group with depressive symptoms $(n=102)$ was composed of individuals who were referred to the clinic because of cognitive complaints (largely memory and concentration difficulties). They endorsed depressive symptoms in the mild depression range or higher on the Beck Depression Inventory (BDI; score $>9)^{22}$ and did not have a prescription for a cholinesterase inhibitor or memantine (Namenda). The patients were primarily taking medications for management of cholesterol and blood pressure. Exclusion criteria for this group with depressive symptoms included a diagnosis at presentation or follow-up of a major neurocognitive disorder, ${ }^{23}$ a neurological disorder (e.g. epilepsy, Korsakoff syndrome, obstructive hydrocephalus, cerebral palsy), or mild cognitive impairment, ${ }^{24}$ except when the mild cognitive impairment criteria was fulfilled but depressive symptomatology was deemed the primary explanatory factor by the clinicians (SP and EF). Patients were also excluded when basic laboratory tests results indicated treatable disorders underlying their cognitive complaints (e.g. low vitamin $\mathrm{B}_{12}$, hypothyroidism). Chart review of the clinical consultation revealed that a history of emotional dysfunction was reported by $31.5 \%$ of these patients, largely depression and/or anxiety symptoms $(26.5 \%)$, and to a lesser extent patients reported panic disorder (2\%), bipolar disorder (1\%), and posttraumatic stress disorder (2\%).

A majority of the group with depressive symptoms attended the clinic once $(72.5 \%)$. A total of $43.1 \%$ of these patients were scheduled for a follow-up visit to track any cognitive changes, but only $39.5 \%$ returned. Of those returning ( 28 patients), $39.2 \%$ were seen once, with an average follow-up time between first and last clinic visits of 6 months; $17.9 \%$ were seen twice, with an average follow-up time of 18 months; and $42.9 \%$ were seen three or more times, with an average follow-up time of 29 months. Of the 28 patients followed, two were eventually diagnosed with Alzheimer's disease after approximately 3 years of follow-up. After the initial consultation, $56.9 \%$ of patients with depressive symptoms were not scheduled for follow-up because depressive symptoms were considered essential/primary to their cognitive status; thus, they were recommended to follow-up with their primary care physicians for treatment (either directly or through psychological/ psychiatric referral), and to be re-referred should cognitive complaints persist.

Alzheimer's disease was diagnosed according to the National Institute of Neurological and Communicative Disorders and Stroke and the Alzheimer's disease and Related Disorders Association $(n=137) .{ }^{25}$ FTD patients met Neary et $\mathrm{al}^{26}$ criteria and included those presenting with behavior $(n=46)$ or language symptoms (progressive nonfluent aphasia, $\mathrm{n}=12$; semantic dementia, $n=9)$. Study exclusion for the dementia groups included any history of traumatic brain injury, stroke, tumor or other neurologic or psychiatric disorder that could account for their symptoms.

Healthy volunteers $(n=41)$ included individuals recruited from the community through posters and newspaper advertisements, caregiver support groups, or approached while accompanying friends or family to clinic visits. They were included if they reported general good physical and cognitive health, and excluded if they reported medical, psychological, or motor conditions that impacted their activities of daily living or their performance on cognitive tests. They reported no cognitive complaints or changes more than what is typical for normal aging relative to their peers and did not have a prescription for a

Table 1: Demographics, BDI ratings, and MoCA scores by group

\begin{tabular}{|c|c|c|c|c|c|c|c|c|}
\hline & $\mathbf{n}$ & Gender M:F & Age* M (SD) & Years education $^{\dagger} \mathrm{M}(\mathrm{SD})$ & Years ill M (SD) & BDI $^{\ddagger} \mathbf{M}(\mathrm{SD})$ & $\mathrm{MoCA}^{\S} \mathrm{M}(\mathrm{SD})$ & $\begin{array}{l}\text { Percentage scoring below } \\
\text { normal MoCA cutoff }(<26)\end{array}$ \\
\hline Dep & 102 & $46: 56$ & $54.00(10.84)$ & $13.53(3.21)$ & $2.69(2.23)$ & $22.31(8.80)$ & $25.05(3.29)$ & 50.0 \\
\hline $\mathrm{AD}$ & 137 & $63: 74$ & 71.93 (10.29) & $9.77(5.38)$ & $3.69(2.42)$ & $7.52(8.08)$ & $14.47(5.68)$ & 99.3 \\
\hline FTD & 67 & $39: 28$ & $67.00(8.64)$ & $13.33(3.15)$ & $4.88(2.79)$ & $9.63(8.98)$ & $17.33(6.96)$ & 89.6 \\
\hline $\mathrm{HC}$ & 41 & $15: 26$ & 58.29 (11.87) & $14.62(2.67)$ & - & $3.39(5.16)$ & 27.59 (1.69) & 17.1 \\
\hline
\end{tabular}

$\mathrm{AD}=$ Alzheimer's disease $; \mathrm{BDI}=$ Beck Depression Inventory; Dep = group with depressive symptoms; $\mathrm{F}=$ female; FTD = frontotemporal dementia;

$\mathrm{HC}=$ healthy control; $\mathrm{M}=$ male; MoCA = Montreal Cognitive Assessment $\mathrm{SD}=$ standard deviation.

*Dep, $\mathrm{HC}<\mathrm{FTD}<\mathrm{AD} ; \mathrm{p}<0.05$ for all using one-way analysis of variance.

${ }^{\dagger}$ Dep, HC, FTD $>$ AD; $p<0.05$ for all using one-way analysis of variance.

${ }^{\ddagger}$ Dep $>$ AD, FTD , HC; FTD $>$ HC; $p<0.05$ for all using one-way analysis of variance.

${ }^{\S} \mathrm{HC}>$ Dep $>$ FTD $>$ AD, $p<0.05$ for all using one-way analysis of variance. 


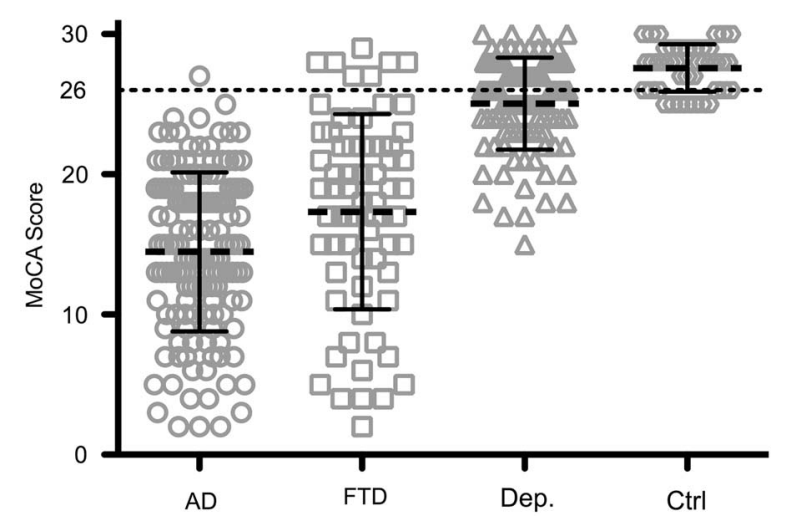

Figure 1: Mean and individual data points on the Montreal Cognitive Assessment (MoCA) for each group $(A D=$ Alzheimer's disease; $F T D=$ frontotemporal dementia; Depressive = Group with depressive symptoms; Controls $=$ healthy controls).

cholinesterase inhibitor or memantine (Namenda). In addition, they were not recruited on the basis of normative cutoffs on a cognitive or mood measure.

\section{Procedures ANd Materials}

All participants completed the MoCA (with education correction applied ${ }^{1}$ ) and the BDI. ${ }^{22}$ Additional cognitive screening measures administered to all participants as part of a standard brief cognitive battery at our clinic were: Mini-Mental State Examination, ${ }^{27}$ Rivermead Behavioral Memory Test Prose Recall Passage, ${ }^{28}$ Trail Making Test, ${ }^{29}$ verbal fluency (FAS and animals), ${ }^{29}$ the Western Aphasia Battery Naming subtest, ${ }^{30}$ and the Clock Drawing Test. ${ }^{31}$

\section{RESULTS}

MoCA scores for the group with depressive symptoms and patients with Alzheimer's disease and FTD are shown in Table 1 and Figure 1. As indicated in Table 1, half of the group with depressive symptoms scored below the suggested normal MoCA cutoff of 26. Across the groups, mean MoCA scores were significantly different using a one-way analysis of variance (ANOVA), $F(3,343)=124.82, \mathrm{p}<.05, \eta_{p}^{2}=.52$ (Figure 1). Post-hoc analyses revealed that as a group, those with depressive symptoms (mean $[\mathrm{M}]=25.05$, standard deviation $[\mathrm{SD}]=3.29$ ) scored significantly lower than healthy controls $(M=27.59$, $\mathrm{SD}=1.69), p<.05$, but significantly higher than the Alzheimer's $(\mathrm{M}=14.47, \mathrm{SD}=5.68)$ and FTD groups $(\mathrm{M}=17.33, \mathrm{SD}=6.96)$, $\mathrm{p}<.05$ for all (Bonferroni correction applied; Table 1). Because education is known to influence MoCA scores, ${ }^{1}$ we examined the impact of education on group comparisons by removing all participants with lower education $(<10$ years of education) and re-running the analyses (20\% of participants removed). The ANOVA results obtained were unchanged and Bonferroni corrected post hoc contrasts showed a marginal difference between those with depressive symptoms and healthy controls, $\mathrm{p}=0.1$.

To examine cognitive domains affected in those with depressive symptoms, we compared their MoCA subtask scores to healthy controls using independent samples $t$-tests. These analyses demonstrated that participants with depressive symptoms scored significantly lower than healthy controls on visuospatial/ executive, repetition, and fluency components of the MoCA, $\mathrm{p}<0.005$ for all (Bonferroni correction applied; Figure 2). These results remained unchanged when participants with lower education were removed $(<10$ years of education; $9 \%$ of participants removed), $\mathrm{p}<0.005$ for all (Bonferroni correction applied). Analyses on supplementary cognitive screens revealed that

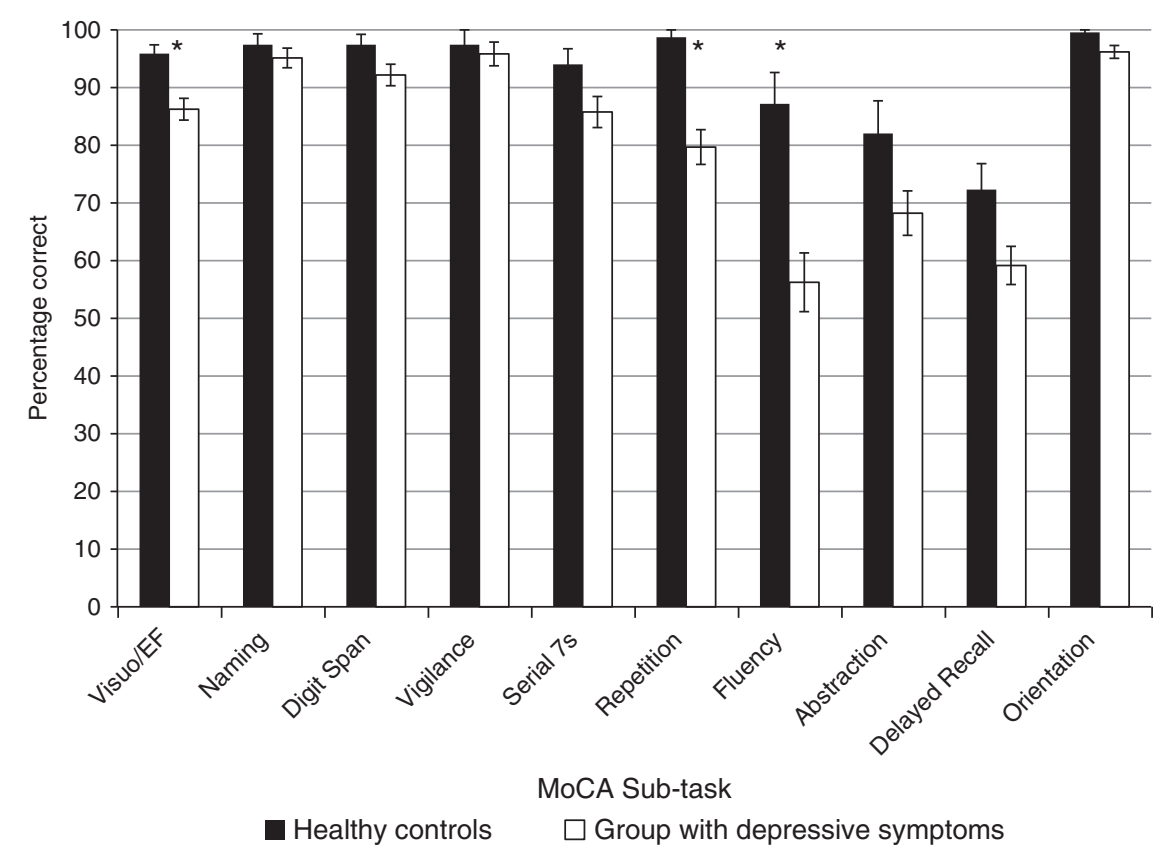

Figure 2: MoCA sub-task performance (mean percentage and standard error bars) of the group with depressive symptoms and healthy controls (Visuo/EF $=$ visuospatial/executive function; * significant group differences). 


\section{Table 2: Cognitive test scores showing means and standard deviations}

\begin{tabular}{|c|c|c|c|c|c|c|c|c|c|c|}
\hline & MMSE & $\begin{array}{l}\text { Prose recall: } \\
\text { immediate }\end{array}$ & $\begin{array}{c}\text { Prose recall: } \\
\text { delay* }^{*}\end{array}$ & $\begin{array}{c}\text { Clock: } \\
\text { command* }\end{array}$ & $\begin{array}{l}\text { Clock: } \\
\text { copy }\end{array}$ & $\begin{array}{c}\text { Phonemic } \\
\text { fluency* }\end{array}$ & $\begin{array}{l}\text { Semantic } \\
\text { fluency* }\end{array}$ & $\begin{array}{c}\text { WAB } \\
\text { Naming }\end{array}$ & TMT A* & TMT B* \\
\hline Dep & $27.82(2.11)$ & $8.64(3.26)$ & $7.47(3.34)$ & $9.25(0.89)$ & $9.63(0.58)$ & $30.59(9.65)$ & $15.86(5.12)$ & $19.60(1.36)$ & $76.84(84.67)$ & $105.21(57.34)$ \\
\hline $\mathrm{HC}$ & $28.71(1.40)$ & $10.19(3.35)$ & $9.33(3.54)$ & $9.85(0.43)$ & $9.82(0.45)$ & $43.51(11.16)$ & $20.49(5.09)$ & $19.97(0.16)$ & $27.10(7.01)$ & $61.72(21.32)$ \\
\hline
\end{tabular}

Highest possible scores: $\mathrm{MMSE}=30$; prose recall - immediate $=21$; prose recall - delay $=21$; clock - command $=10$; clock - copy $=10$; WAB naming = 20; TMT A and B discontinued after 300 seconds.

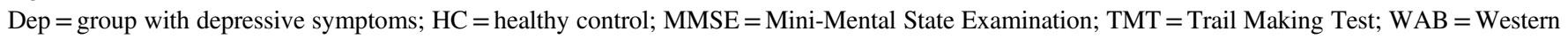
Aphasia Battery.

*Significant results using independent samples $t$-tests, $\mathrm{p}<.005$ (Bonferroni corrected).

healthy controls scored significantly better than patients with depressive symptoms on delayed prose recall, Clock Drawing Test, verbal fluency (FAS and animals), and the Trail Making Test, $\mathrm{p}<0.005$ for all (Table 2; Bonferroni correction applied).

\section{DISCUSSION}

In this study, we examined the influence of mood symptoms on MoCA performance in a memory clinic sample of patients endorsing depressive symptoms. Half of the group with depressive symptoms scored below the MoCA-suggested cutoff for cognitive impairment. As a group, these individuals scored below healthy controls, but above those with Alzheimer's disease and frontotemporal dementia. In addition, MoCA subtask analysis revealed a pattern of executive/attentional dysfunction in the group endorsing depressive symptoms.

The findings of this study demonstrate the susceptibility of individuals with depressive symptoms to obtain impaired scores on the MoCA, not unlike other cognitive screens (prose recall, clock drawing, verbal fluency, and trail making) (see Musso et al for similar MoCA findings in severe mental illness impacting $\operatorname{mood}^{32}$ ). Thus, these results provide an important caveat/potential explanatory factor for clinicians when using the MoCA to interpret a patient's global cognition. In a memory clinic setting, with cognitive impairment indicated by the MoCA in the context of depressive symptoms, differential diagnosis may be aided by detailed assessment of functional capacities, as well as cognitive investigations with other domain-specific screens or in-depth neuropsychological evaluation. Such additional testing often reveals more severe cognitive deficits across various domains in those with dementia relative to individuals with depressive symptoms. ${ }^{33,34}$ Additionally, objective cognitive impairment in those presenting with depressive symptoms necessitates pharmacological or psychotherapeutic intervention; however, cognitive benefits may potentially be moderated by underlying neurodegenerative pathology.

Together with global cognitive decline, deficits in a range of cognitive domains are associated with mood dysfunction, especially in those with late-life onset of mood symptoms (age 50-65 years). ${ }^{35}$ In the present study, individuals with depressive symptoms showed an executive/attentional pattern of weakness relative to healthy controls on the MoCA, consistent with a recent meta-analysis showing the impact of mood dysfunction on multiple executive/attentional domains. ${ }^{36}$ Moreover, these observed executive/attention deficits are in line with theories of mood dysfunction that posit dysregulation in prefrontal regions (asymmetric ventromedial vs dorsolateral function $)^{37}$ and cortico-striatal-pallidal-thalamic circuits (dysfunction in prefrontal subregions and anterior cingulate cortex connections with basal ganglia and thalamic structures). ${ }^{38,39}$

A strength of this work is the observed influence of mood symptoms on the MoCA in a large cohort of patients with depressive symptoms in a clinical setting, unlike research thus far that primarily involves community samples. Nevertheless, this work is limited by the inclusion of patients with depressive symptoms who presented with subjective cognitive complaints, the majority of whom were without a psychiatric diagnosis at the time of consultation. Thus, research is needed to clarify whether our findings extend to individuals with a diagnosed mood disorder. Of the $40 \%$ of these individuals with depressive symptoms who returned for follow-up, only two eventually met criteria for Alzheimer's disease, suggesting that the majority of these patients did not progress to a neurodegenerative disease. A longer longitudinal follow-up may be necessary to clarify whether the group with depressive symptoms will eventually develop neurodegenerative disease.

Moreover, although we used of a healthy control comparison group, it might be argued than a better control group would include patients presenting to the clinic with similar characteristics as our group with depressive symptoms (cognitive complaints, same inclusion and exclusion criteria), but who do not meet criteria for depressive symptomatology $(B D I<10)$. Notably, these patients $(n=40$; retrieved from our clinic database) had similar mean MoCA scores as our group with depressive symptoms $(p>0.05)$ and $43 \%$ fell below MoCA cutoff (lower than the $50 \%$ in the group with depressive symptoms). Although these patients did not meet BDI criteria for depressive symptoms, they represent a questionable control group in a memory clinic setting because depressive symptomatology cannot be ruled out in these patients; $23 \%$ reported either a history of depression or stated at least two symptoms that comprise a major depressive episode according to Diagnostic and Statistical Manual of Mental Disorders IV criteria. $^{40}$ Furthermore, as the BDI threshold for depressive symptoms is likely variable depending on the medical population studied, ${ }^{41}$ future work with patients diagnosed by Diagnostic and Statistical Manual of Mental Disorders criteria will help to further clarify the sensitivity of the MoCA to depressive symptomatology, an approach not typically afforded to memory clinics. 
In conclusion, individuals endorsing depressive symptoms often demonstrate scores on the MoCA that fall below the normal cutoff. Consequently, mood status, documented through routine mood screening, should be considered an important caveat when interpreting MoCA scores. Longitudinal research, with the involvement of early neuropathological biomarkers, is necessary to better inform this mood-cognition relationship and the MoCA's prognostic value to differential diagnosis in patients presenting depressive symptoms.

\section{Disclosures}

The authors have nothing to disclose.

\section{REFERENCES}

1. Nasreddine ZS, Phillips NA, Bedirian V, et al. The Montreal Cognitive Assessment, MoCA: a brief screening tool for mild cognitive impairment. J Am Geriatr Soc. 2005;53:695-9.

2. Julayanont P, Phillips N, Chertkow H, Nasreddine ZS. Montreal Cognitive Assessment (MoCA): concept and clinical review. In: Larner AJ, ed. Cognitive screening instruments: a practical approach. London: Springer-Verlag; 2013:111-52.

3. Benedetti F, Bernasconi A, Pontiggia A. Depression and neurological disorders. Curr Opin Psychiatry. 2006;19:14-8.

4. Raskind MA. Diagnosis and treatment of depression comorbid with neurologic disorders. Am J Med. 2008;121(Suppl 2):S28-37.

5. Rickards H. Depression in neurological disorders: an update. Curr Opin Psychiatry. 2006;19:294-8.

6. Knapskog AB, Barca ML, Engedal K. Prevalence of depression among memory clinic patients as measured by the Cornell Scale of Depression in Dementia. Aging Ment Health. 2014; 18:579-87.

7. Gao Y, Huang C, Zhao K, Ma L, et al. Depression as a risk factor for dementia and mild cognitive impairment: a meta-analysis of longitudinal studies. Int J Geriatr Psychiatry. 2013;28:441-9.

8. Green RC, Cupples LA, Kurz A, et al. Depression as a risk factor for Alzheimer disease: the MIRAGE Study. Arch Neurol. 2003; 60:753-9.

9. Ritchie K, Gilham C, Ledésert B, Touchon J, Kotzki PO. Depressive illness, depressive symptomatology and regional cerebral blood flow in elderly people with sub-clinical cognitive impairment. Age Ageing. 1999;28:385-91.

10. Steffens DC, Otey E, Alexopoulos GS, et al. Perspectives on depression, mild cognitive impairment, and cognitive decline. Arch Gen Psychiatry. 2006;63:130-8.

11. Wilson RS, Barnes LL, Mendes de Leon CF, et al. Depressive symptoms, cognitive decline, and risk of $\mathrm{AD}$ in older persons. Neurology. 2002;59:364-70.

12. Drevets WC. Neuroimaging studies of mood disorders. Biol Psychiatry. 2000;48:813-29.

13. Sapolsky RM, Krey LC, McEwen BS. Prolonged glucocorticoid exposure reduces hippocampal neuron number: Implications for aging. J Neurosci. 1985;5:1222-7.

14. Sheline YI. Neuroimaging studies of mood disorder effects on the brain. Biol Psychiatry. 2003;1(54):338-52.

15. Ganguli M, Du Y, Dodge $\mathrm{HH}$, Ratcliff GG, Chang $\mathrm{CH}$. Depressive symptoms and cognitive decline in late life.A prospective epidemiological study. Arch Gen Psychiatry. 2006; 63:153-60.

16. Dotson VM, Davatzikos C, Kraut MA, Resnick SM. Depressive symptoms and brain volumes in older adults: a longitudinal magnetic resonance imaging study. J Psychiatry Neurosci. 2009;34:367-75.

17. Levin RL, Heller W, Mohanty A, Herrington JD, Miller GA. Cognitive deficits in depression and functional specificity of regional brain activity. Cogn Ther Res. 2007;31:211-33.

18. Royall DR, Palmer R, Chiodo LK, Polk MJ. Depressive symptoms predict longitudinal change in executive control but not memory. Int J Geriatr Psychiatry. 2012;27:89-96.
19. Baer LH, Tabri N, Blair M, Bye D, Li KZ, Pushkar D. Longitudinal associations of need for cognition, cognitive activity, and depressive symptomatology with cognitive function in recent retirees. J Gerontol B Psychol Sci Soc Sci. 2013;68:655-4.

20. Del Brutto OH, Mera RM, Del Brutto VJ, et al. Influence of depression, anxiety and stress on cognitive performance in community-dwelling older adults living in rural Ecuador: results of the Atahualpa Project. Geriatr Gerontol Int. 2015;15:508-14.

21. Freitas S, Simões MR, Alves L, Santana I. Montreal Cognitive Assessment: influence of sociodemographic and health variables. Arch Clin Neuropsychol. 2012;27:165-75.

22. Beck AT, Ward CH, Mendelson M, Mock J, Erbaugh J. An inventory for measuring depression. Arch Gen Psychiatry. 1961;4:561-71.

23. American Psychiatric Association. Diagnostic and Statistical Manual of Mental Disorders: DSM-5. Arlington, VA: American Psychiatric Association; 2013.

24. Winblad B, Palmer K, Kivipelto M, et al. Mild cognitive impairment -beyond controversies, towards a consensus: report of the International Working Group on Mild Cognitive Impairment. J Intern Med. 2004;256:240-6.

25. McKhann G, Drachman D, Folstein M, et al. Clinical diagnosis of Alzheimer's disease: report of NINCDS-ADRDAWork Group under auspices of Department of Health and Human Services Task Force on Alzheimer's Disease. Neurology. 1984;34:939-44.

26. Neary D, Snowden JS, Gustafson L, et al. Frontotemporal lobar degeneration - a consensus on clinical diagnostic criteria. Neurology. 1998;51:1546-54

27. Folstein MF, Folstein SE, McHugh PR. "Mini-mental state:" a practical method for grading the cognitive state of patients for the clinician". J Psychiatr Res. 1975;12:189-98.

28. Wilson B, Cockburn J, Baddeley AD. The Rivermead behavioural memory test. Bury St. Edmunds. Suffolk: Thames Valley Test Company; 1991.

29. Strauss E, Sherman EM, Spreen O. A compendium of neuropsychological tests: administration, norms, and commentary. New York: Oxford University Press; 2006.

30. Kertesz A. The Western Aphasia battery. New York, NY: Grune \& Stratton; 1982.

31. Rouleau I, Salmon DP, Butters N, et al. Quantitative and qualitative analyses of clock drawings in Alzheimer's and Huntington's disease. Brain Cogn. 1992;18:70-87.

32. Musso MW, Cohen AS, Auster TL, et al. Investigation of the Montreal Cognitive Assessment (MoCA) as a cognitive screener in severe mental illness. Psych Res. 2014;220:664-8.

33. Christensen H, Griffiths K, MacKinnon A, Jacomb P. A quantitative review of cognitive deficits in depression and Alzheimer-type dementia. J Int Neuropsychol Soc. 1997;3:631-51.

34. Wright SL, Persad C. Distinguishing between depression and dementia in older persons: neuropsychological and neuropathological correlates. J Geriatr Psychiatry Neurol. 2007;20:189-98.

35. Herrmann LL, Goodwin GM, Ebmeier KP. The cognitive neuropsychology of depression in the elderly. Psychol Med. 2007;37:1693-702.

36. Snyder HR. Major depressive disorder is associated with broad impairments on neuropsychological measures of executive function: a meta-analysis and review. Psychol Bull. 2013;139: $81-132$.

37. Koenigs M, Grafman J. The functional neuroanatomy of depression: distinct roles for ventromedial and dorsolateral prefrontal cortex. Behav Brain Res. 2009;201:239-43.

38. Bora E, Harrison BJ, Davey CG, Yücel M, Pantelis C. Meta-analysis of volumetric abnormalities in cortico-striatal-pallidal-thalamic circuits in major depressive disorder. Psychol Med. 2012;42: 671-81.

39. Price JL, Drevets WC. Neurocircuitry of mood disorders. Neuropsychopharmacology. 2010;35:192-216.

40. American Psychiatric Association. Diagnostic and statistical manual of mental disorders: DSM-IV-TR. Washington, DC: American Psychiatric Association; 2000.

41. Wang Y-P, Gorenstein C. Assessment of depression in medical patients: a systematic review of the utility of the Beck Depression Inventory-II. Clinics. 2013;68:1274-87. 